\title{
Cenários experimentais: efeito sobre a emoção e o comportamento pró-social
}

\author{
Ronaldo Pilati \\ Universidade de Brasília
}

\begin{abstract}
Resumo
Emoções antecedentes ao comportamento pró-social são influenciadas pela forma como os eventos são interpretados. Estudos têm demonstrado que a atribuição de incontrolabilidade do comportamento de alguém está associada à produção de estados afetivos de simpatia ou pena. Esta asserção teórica tem sido corroborada por meio do uso de cenários de simulação experimental. O objetivo deste estudo é avaliar o efeito que a variação dos cenários, como a proximidade social do protagonista e as normas sociais subjacentes ao cenário, possuem sobre a relação atribuição-ajuda. Foi desenvolvido um estudo com 24 cenários do qual participaram 547 estudantes universitários. Os resultados indicaram que, independentemente do cenário, a percepção de incontrolabilidade levou a emoções simpáticas (ou de compaixão) que, por sua vez, estão associadas a uma maior intenção de ajuda. Além disto, cenários que emulam normas de reciprocidade estão associados à maior intenção de ajuda.
\end{abstract}

Palavras-Chave: comportamento pró-social; simpatia; simulação experimental

\begin{abstract}
Experimental simulations: effect over emotions and prosocial behavior. Prosocial behavior emotions are influenced by how events are interpreted. Studies have shown that the attribution of incontrolabitily to one's behavior is associated with the production of sympathy emotions. This theoretical assertion has been corroborated with experimental scenarios. This study aimed to evaluate the effect of variations of the scenarios, such as the social proximity of the protagonist and social norms have over prosocial behavior intentions. An experimental study with 24 scenarios was conducted and 547 university students took part. The results indicate that regardless of the scenario content perceived misfortune leads to sympathy or pity emotions which, in turn, are associated with greater helping intention. Reciprocity norm scenarios were associated with greater helping intentions.
\end{abstract}

Keywords: prosocial behavior; sympathy; experimental simulation

$\mathrm{O}$ estudo do comportamento pró-social continua sendo foco de atenção da pesquisa em psicologia social desde a década de 1960. Atualmente os psicólogos sociais têm se interessado em avançar a pesquisa sobre os múltiplos fatores que determinam este tipo de conduta humana, considerando aspectos de diversos níveis de análise como seus antecedentes. A compreensão teórica destes antecedentes auxilia no desenvolvimento de políticas públicas e ações planejadas para produzir uma vida melhor em sociedade, proporcionando maior civilidade e cooperação nas relações sociais. Publicações recentes demonstram este interesse, procurando sistematizar o conhecimento já produzido sobre os antecedentes e consequentes do comportamento pró-social, bem como avançar na compreensão teórica do fenômeno (Mikulincer \& Shaver, 2010; Stürmer \& Snyder, 2010a).

Um dos processos relevantes para a compreensão das emoções simpáticas antecedentes do comportamento pró-social está alicerçada no modelo teórico da atribuição-emoção-ajuda. A influência dos processos cognitivos de atribuição de causalidade tem sido estudada em psicologia social como um dos fatores antecedentes da produção de estados afetivos motivacionais da conduta pró-social. Este trabalho tem como objetivo testar a influência da variação de conteúdos de cenários experimentais (por exemplo, nível de proximidade social do protagonista do cenário com o participante da pesquisa (por exemplo observador) e padrões de conduta - normas sociais - esperados nas situações emuladas nos cenários) na relação mediada de cognição-emoçãointenção de ajuda. A pesquisa que testou o modelo atribuiçãoemoção-ajuda foi realizada por meio de um cenário experimental que emula uma situação de empréstimo de anotações de sala de aula, típico do ambiente universitário. A proposta deste trabalho é diversificar os cenários experimentais, como forma de contribuir para o teste do modelo teórico, variando o tema e a situação dos cenários, mas mantendo a manipulação das variáveis independentes (por exemplo, controlabilidade da situação por parte do protagonista do cenário e custo pessoal 
da ajuda). Desta forma, espera-se produzir evidências de que o processo psicológico preconizado pelo modelo também seja observado em cenários de simulação experimental diferentes daquele utilizado na pesquisa prévia.

O conjunto de antecedentes do comportamento prósocial é bastante abrangente e recentemente a literatura da área tem buscado compreendê-los em termos de múltiplos níveis, considerando antecedentes do micronível, mesonível e macronível de análise (Dovidio, Piliavin, Schroeder, \& Penner, 2006; Penner, Dovidio, Piliavin, \& Schroeder, 2005). A pesquisa no micro e mesonível tem sido intensa nos últimos anos, considerando, por exemplo, fatores evolutivos para a produção de empatia (de Waal, 2008; Sampaio, Camino, \& Roazzi, 2009), o funcionamento do cérebro em estados emocionais em situações de conduta pró-social (Hein \& Singer, 2010) ou os fatores situacionais como desencadeadores de estados emocionais antecedentes do comportamento pró-social (Batson, van Lange, Ahmad, \& Lishner, 2003). Os aspectos emocionais têm sido investigados como um conjunto relevante de antecedentes do comportamento pró-social no micronível e no mesonível de análise. $\mathrm{O}$ presente trabalho foca-se no estudo dos aspectos situacionais, relativos ao mesonível de análise, desencadeadores de estados emocionais antecedentes da conduta pró-social.

No concernente à discussão conceitual sobre tais estados emocionais vale a apresentação de conceitos correlatos ao que foi testado no presente estudo. $\mathrm{O}$ mais pronunciado dos conceitos neste segmento é, provavelmente, a empatia. Não existe, propriamente, uma definição consensual de empatia em psicologia social, muitas vezes ocorrendo sobreposições com conceitos correlatos, como simpatia e compaixão (Sampaio et al., 2009). Por exemplo, Batson (2010), em sua hipótese da empatia-altruísmo, define como conceito central a preocupação empática (empathic concern) que é definida como "uma resposta emocional dirigida à outra pessoa eliciada por e congruente com o bem estar percebido de uma pessoa em necessidade" (p. 20). O autor argumenta que a preocupação empática inclui sentimentos de simpatia, compaixão e ternura e, portanto, não deve ser confundida com a habilidade cognitiva de perceber corretamente o estado emocional interno de outra pessoa. A definição de preocupação empática coloca o estado emocional claramente como uma resposta a um estímulo social situacional. Esta definição, fruto de uma linha de mais de três décadas de investigação do referido autor, é bastante coerente com uma perspectiva situacionalista de estudos do comportamento pró-social, algo recorrente na literatura de psicologia social e classificado como pertencente a antecedentes do mesonível de análise (Penner et al., 2005).

Por outro lado a empatia pode ser definida como um traço ou habilidade com característica de estabilidade, fruto, por exemplo, do desenvolvimento psicológico de um indivíduo. Eisenberg (2010) define empatia como "uma resposta afetiva decorrente da apreensão ou compreensão do estado ou condição emocional de outra pessoa" (p. 130). Esta concepção mais disposicional da empatia tem sido largamente empregada na pesquisa sobre o fenômeno e, usualmente, tem sido operacionalizada por meio de inventários e escalas psicométricas para a mensuração do construto (Falcone et al., 2008; Koller, Camino, \& Ribeiro,
2001). Não há motivos para se negar que respostas emocionais situacionais tenham relação com traços disposicionais e, certamente, traços empáticos têm uma influência significativa na reação emocional decorrente da busca por redução da angústia pessoal (personal distress) ou de sentimentos de simpatia. De qualquer sorte, não é objetivo do presente trabalho propor soluções conceituais para a rede de conceitos relacionados às emoções ou sentimentos antecedentes do comportamento prósocial, muito menos testar um modelo de moderação de traços disposicionais e fatores situacionais como antecedentes do comportamento pró-social. O presente estudo tão somente se propõe a avaliar o efeito de fatores situacionais sobre a relação entre emoções simpáticas e a intenção de ajuda.

O presente trabalho repousa sobre uma tradição de estudos do comportamento pró-social na psicologia social que busca compreender os antecedentes situacionais de estados emocionais, mais especificamente alicerçado nas teorias de atribuição de causalidade (Dela-Coleta \& Dela-Coleta, 2006; Weiner, 1995), como um aporte para se compreender como este processo promove sentimentos de simpatia que motivam o comportamento pró-social. Neste sentido concebe-se que as emoções antecedentes, manipuladas e mensuradas no presente trabalho, dizem respeito a sentimentos de simpatia ou compaixão da pessoa que necessita de ajuda. Conforme preconizado pelo modelo teórico em tela (Weiner, 1995) o processo de julgamento de responsabilidade está associado à produção de sentimentos que funcionam como motivações para o comportamento prósocial. Estas emoções são claramente circunscritas ao que, na literatura de psicologia social, tem sido nomeada de simpatia, aproximando-se da conceituação proposta por Batson (2010) para a preocupação empática. Portanto, não se trata de um traço ou disposição empática, mas sim de um estado afetivo provocado por um fator situacional.

Os processos de mediação cognitiva sobre os estados emocionais antecedentes do comportamento pró-social têm sido investigados na literatura de psicologia social ao menos desde a década de 1970. Um modelo influente na literatura sobre a relação entre cognição-emoção-ajuda foi apresentado por Weiner (1980, 1995). Neste modelo o processo de atribuição de causalidade funciona como um mecanismo que aciona a reação emocional motivadora do comportamento. $\mathrm{O}$ autor postula que quando um observador percebe que a pessoa em necessidade de ajuda possui controle sobre a situação que a levou à solicitação, a intenção de ajuda é menor. Isso ocorre porque este observador tem reações afetivas de culpabilização da vítima e desidentificação, já que o solicitante da ajuda poderia ter evitado chegar àquela situação. Por outro lado, quando a percepção é de impossibilidade de controle, ou seja, de que o solicitante não teve como evitar chegar à situação que o levou a pedir auxílio, a intenção de ajuda do observador é maior, porque esta percepção levaria à geração de sentimentos de simpatia e compaixão com o solicitante. Para testar sua elaboração teórica, Weiner realizou um estudo de simulação experimental com estudantes universitários, no qual as emoções foram mensuradas e a atribuição de controle manipulada em cenários experimentais. Weiner apresentou evidências de que o grau de controle da situação, atribuída pelo observador ao comportamento do solicitante, medeia o tipo de 
reação emocional, que por sua vez está associada ao aumento da probabilidade em ajudar. O modelo teórico testado pelo autor recebeu suporte empírico de estudos posteriores (Betancourt, 1990; Reisenzein, 1986; Rodrigues \& Assmar, 2003; Schmidt \& Weiner, 1988). Entretanto, apesar das evidências favoráveis, o que se nota é que o tipo de cenário experimental utilizado para o teste do modelo é consideravelmente restrito, focado em situações corriqueiras no ambiente universitário. Como forma de ampliar o teste deste modelo teórico o presente trabalho visa ampliar as situações e características dos cenários, expandindo-os para além do ambiente universitário e manipulando elementos de normas e proximidade social do protagonista do cenário com o observador (por exemplo, participante da pesquisa). Desta forma espera-se ampliar a compreensão do modelo teórico e testar se o mesmo funciona da forma preconizada também em diferentes situações sociais emuladas.

A literatura de psicologia social tem apontado que o custo pessoal em ajudar é outro fator antecedente relevante do comportamento pró-social (Batson, 1998; Batson et al., 2003; Dovidio \& Penner, 2001). Neste sentido a inserção de variações do custo pessoal em cenários experimentais é um elemento relevante para a criação de cenários mais aproximados a uma situação real de comportamento, emulando fatores relevantes para o processo de tomada de decisões. Em geral a hipótese do custo pessoal é que quanto maior for este menor a chance de ajuda (Batson, 1998). Esta variável apresentou efeito interativo significativo com a variação da atribuição de responsabilidade do comportamento do indivíduo protagonista em cenários de simulação experimental, tendo a hipótese do custo pessoal recebido apoio empírico (Pilati, Leão, Vieira, \& Fonseca, 2008).

Uma questão importante suscitada pelos estudos que buscaram a evidência desta relação de mediação atribuiçãoemoção-ajuda diz respeito à estratégia metodológica de simulações experimentais (Weiner, 1980). O conteúdo do cenário e o tipo de situação eliciada podem ser fatores relevantes para a determinação da intenção de ajuda. Alguns dos fatores que podem exercer influência sobre a relação hipotetizada pelo modelo de Weiner dizem respeito, por exemplo, ao nível de proximidade do participante de quem solicita ajuda (por exemplo, um amigo ou um desconhecido em uma fila) e as normas sociais indicadoras de como se comportar em determinadas situações sociais emuladas nos cenários experimentais.

Sobre a proximidade social do solicitante da ajuda em relação ao observador, a literatura de psicologia social tem demonstrado sistematicamente que a forma como os indivíduos categorizam aqueles com os quais entram em contato é importante na decisão em ajudar (Stürmer \& Snyder, 2010a; Stürmer, Snyder, Krop, \& Siem, 2006; Stürmer, Snyder, \& Omoto, 2005). A pesquisa sistemática tem demonstrado que existe um favoritismo do endogrupo, o que levaria a se supor que um cenário em que o indivíduo que demanda ajuda tem proximidade com o participante eliciaria maior intenção de ajuda. Com esta suposição, cenários que apresentam uma pessoa mais próxima (um amigo, parente ou indivíduo que pertença a um mesmo grupo de categorização social) levariam o participante a avaliar como mais provável a intenção de ajudar o solicitante.
A literatura de psicologia social tem demonstrado que as normas sociais são indutoras importantes de comportamento em diferentes contextos experimentais (Aarts, Dijkesterhuis, \& Custers, 2003; Cialdini, Reno, \& Kallgren, 1990; Macrae \& Johnston, 1998), seja por sua ativação automática ou controlada. As normas sociais também são antecedentes relevantes do comportamento pró-social (Dovidio et al., 2006; Moser \& Corroyer, 2001) e a geração de cenários experimentais que suscitam padrões diferentes de normas sociais podem exercer um efeito relevante sobre as predições do modelo de Weiner (1980, 1995). Tipicamente o cenário utilizado para o teste do modelo atribuição-emoção-ajuda utiliza uma situação de coleguismo de estudantes de graduação, o que invoca aspectos específicos da norma da reciprocidade. Supõe-se que a variação deste elemento, utilizando-se cenários mais próximos do círculo familiar do participante da pesquisa ou outro que esteja associada à relação com um estranho que usa um serviço público, podem estar associados a diferentes padrões de intenção de ajuda.

Diante do conjunto de antecedentes do comportamento pró-social e da escassez de estudos empíricos que utilizem cenários experimentais variados, isto é, que busquem avaliar a proximidade social do protagonista do cenário com o observador (por exemplo, participante da pesquisa) para o teste do modelo de Weiner (1980), faz-se necessário o desenvolvimento de um estudo que busque variar conteúdos dos cenários. Esta variação dos cenários considera os seguintes elementos: (1) a categorização social do indivíduo que solicita ajuda, tornando-o mais próximo ou distante do observado; e (2) situações sociais diversas que emulem diferentes normas sociais (fila de cartório, amizade com intimidade). Desta forma é possível testar diferencialmente o grau de estabilidade das predições teóricas do modelo atribuição-emoção-ajuda.

\section{Método}

Para se alcançar os objetivos descritos, foi utilizado um estudo de simulação quase-experimental de cenários com delineamento fatorial 4 (Temas: anotações, teatro, fila e aula) $\mathrm{x}$ 2 (atribuição: controlabilidade e incontrolabilidade) x 3 (custo: baixo, médio e alto). Considera-se este estudo como quaseexperimental por não haver garantia de designação aleatória dos participantes para cada uma das condições experimentais (Shadish, Cook, \& Campbell, 2002).

\section{Participantes}

Participaram do estudo 547 estudantes universitários (64,5\% mulheres), convidados a participar voluntariamente em salas de aula de instituições de ensino superior do Distrito Federal. Suas idades variaram entre 16 e 60 anos com média de 23,7 anos (DP =7,6). A mediana da renda familiar mensal foi de R $\$ 5.500,00 \mathrm{e}$ a renda média relatada pelos participantes foi de $\mathrm{R} \$ 15.581,41$ $(D P=139.099,90)$.

\section{Instrumentos}

O instrumento utilizado foi um questionário de uma folha, frente e verso. Este era composto por quatro partes. A primeira continha explicações gerais sobre o projeto de pesquisa e sobre 
o grupo responsável pelo mesmo e o consentimento apresentado e informado, pelo participante, oralmente. A segunda parte continha o texto com a apresentação do cenário experimental e instruções de que o participante deveria considerar apenas as informações contidas na situação apresentada para responder as questões da pesquisa.

A terceira parte do questionário continha: (a) a medida da variável dependente, intenção de ajuda, feita em uma pergunta associada a uma escala Likert com 11 pontos ancorada em 0 (certeza de não ajudar) e 10 (certeza de ajudar); (b) dez questões para checagem da manipulação experimental e mensuração da percepção de controle e sentimentos de simpatia em relação ao sujeito do cenário. Estas questões estavam associadas a uma escala Likert ancorada em sete pontos de concordância. A última parte do questionário era composta por indicadores de caracterização sociodemográfica: sexo, idade, escolaridade e renda mensal. $\mathrm{O}$ instrumento e o procedimento experimental foram submetidos a uma validação semântica e a um teste piloto. Análises fatoriais indicaram que os itens de atribuição de causa ao comportamento se agregaram em um único fator com cargas fatoriais variando de 0,62 a 0,82 e bom índice de consistência interna $(\alpha=0,83)$. Compôs esse fator os itens " $O$ seu colega não é responsável pela situação na qual se encontra; $\mathrm{O}$ seu colega é responsável pela situação na qual se encontra; A necessidade de seu colega pedir ajuda surgiu por uma razão externa a ele; A necessidade de seu colega pedir ajuda surgiu por uma razão interna a ele". Os itens de emoções desencadeadas pela situação se agregaram em um fator com cargas variando entre 0,37 e 0,91 e índice de consistência interna aceitável $(\alpha=0,65)$. Compôs esse fator os itens "Você sente irritação em relação ao seu colega que lhe pede ajuda; Você sente raiva do seu colega que lhe pede ajuda; Você sente simpatia em relação ao seu colega que lhe pede ajuda".

\section{Procedimentos}

A coleta foi realizada em grupos de alunos nas salas de aula, com a anuência do professor responsável. Após a distribuição de um questionário para cada participante, garantindo equilíbrio de participantes em cada condição experimental, foi feita uma apresentação da pesquisa, que consistia em apresentar o aplicador, o grupo responsável pela pesquisa, o tema e o objetivo do estudo. Estas informações estavam padronizadas, descritas na primeira seção do questionário de pesquisa. $\mathrm{O}$ instrumento era apresentado, enfatizando-se que este consistia em frente e verso e que a leitura atenta da história era essencial. Em média os participantes levaram 15 minutos para responder o questionário.

Os cenários criados para este estudo foram adaptados do estudo de Weiner (1980), que trabalhou com apenas um tema de cenário (por exemplo, anotações). Foram elaborados cenários que buscaram manipular o nível de proximidade do solicitante da ajuda e as normas sociais da situação apresentada. A primeira parte dos cenários de um mesmo tema era idêntica, e a variação das outras duas variáveis independentes (VI) foi realizada por meio da diferenciação do desfecho da situação apresentada. $\mathrm{O}$ cenário para o tema anotações foi o seguinte:

Suponha que um colega de sua turma lhe procura e pede suas anotações das aulas da última semana para copiá-las, com o objetivo de preparar-se para a próxima prova.

Finalizações da VI atribuição:

Diz que não anotou nada porque estava viajando a lazer durante a semana anterior.

Diz que não anotou nada na última semana porque estava com uma infecção no olho que lhe impedia de ler as informações anotadas no quadro pelo professor.

Finalizações da VI custo:

Ele diz que só vai gastar dez minutos para lhe devolver as folhas.

Ele diz que só poderá lhe devolver as anotações três dias antes da prova.

Ele diz que só poderá lhe devolver as anotações no dia da prova.

O cenário para o tema teatro foi o seguinte:

Suponha que você encontra um amigo na entrada do teatro. Ele pede $\mathrm{R} \$ 100,00$ emprestados para comprar um novo ingresso.

Finalizações da VI atribuição:

Seu amigo explica que teve a carteira roubada, com o ingresso, cartões e dinheiro enquanto estava a caminho do teatro. Ele the diz que devolverá o dinheiro assim que possível.

Seu amigo explica que deixou a carteira em casa, com o ingresso e dinheiro, quando saiu para o teatro. Ele lhe diz que devolverá o dinheiro assim que possível.

Finalizações da VI custo:

Você tem $\mathrm{R} \$ 120,00$ em sua carteira e poderia emprestar o dinheiro sem prejuízos para você.

Você tem $\mathrm{R} \$ 120,00$ em sua carteira e planejou sair para jantar após o teatro.

Você tem $\mathrm{R} \$ 120,00$ em sua carteira e não tem mais dinheiro para a semana.

O cenário para o tema fila foi o seguinte:

Suponha que você está na fila do cartório, às 8:50, aguardando o número de sua senha chegar e você é o próximo. Um rapaz se aproxima e lhe pede para trocar de senhas com ele. Para a senha dele há 10 pessoas na frente.

Finalizações da VI atribuição:

Ele justifica que precisa realizar a autenticação até às 9:00 e que chegou atrasado porque perdeu a hora de se levantar.

Ele justifica que precisa autenticar um documento até às 9:00 e que chegou atrasado porque os ônibus entraram em greve.

Finalizações da VI custo:

Você tem tempo disponível.

Você tem um compromisso importante e não pode se atrasar.

Você estacionou em local proibido e está com medo de ser multado. 
O cenário para o tema aula foi o seguinte:

Suponha que um casal de amigos lhe peça para dar uma aula particular de matemática para o filho deles hoje à noite, porque amanhã ele terá prova. Você tem os conhecimentos necessários em matemática.

Finalizações da VI atribuição:

Eles justificam que marcaram de ir ao cinema e por isso não podem ajudar o filho.

Eles justificam que não entendem a matéria, e por isso não podem ajudar o filho.

Finalizações da VI custo:

Você tem tempo disponível.

Para ajudá-lo você deixará de assistir a um filme no cinema, que havia programado anteriormente.

Para ajudá-lo você deixará de ir à festa de aniversário de sua mãe.

Do total de participantes 181 responderam cenários do tema anotações, 102 do tema teatro, 135 do tema fila e 129 do tema aula. O número de participantes em cada condição experimental foi equitativo para todos os temas de cenários. Foi realizada análise exploratória para adequação dos dados aos pressupostos do modelo linear geral (Hair, Anderson, Tatham, \& Black, 2005; Tabachnick \& Fidell, 2001). Os dados foram analisados por meio de ANOVA univariada de múltiplas vias e análise de trajetória. A análise de trajetórias foi realizada com o programa AMOS 7.0, utilizando-se o estimador da máxima verossimilhança (MLE) para o cálculo dos coeficientes de regressão do modelo de mediação. O projeto foi submetido e aprovado por um Comitê de Ética em Pesquisa.

\section{Resultados}

Os dados descritivos em relação a todas as condições experimentais e seus respectivos níveis de agregação são apresentados na Tabela 1 .

Como pode ser observado na Tabela 1 houve diferença em relação à intenção de ajuda quando comparados os temas dos cenários $[F(3,527)=10,97 ; p \leq 0,001]$, sendo que os participantes do tema anotações tiveram maior intenção de ajuda. Em relação à variável atribuição também foi observada diferença significativa $[F(1,529)=17,88 ; p \leq 0,001]$ com maior média para o grupo de percepção de incontrolabilidade. Os participantes

Tabela 1

Médias e desvios-padrão (parênteses) da variável dependente ajuda para cada uma das condições experimentais do estudo

\begin{tabular}{|c|c|c|c|c|c|c|c|}
\hline \multirow{2}{*}{$\begin{array}{c}\text { ATRIBUIÇÃO } \\
\text { Incontrolabilidade }\end{array}$} & \multicolumn{3}{|c|}{ CUSTO } & \multicolumn{4}{|c|}{ TEMAS } \\
\hline & $6,6 *(2,8)$ & Baixo & $7,7 *(2,5)$ & Anotações & Teatro & Fila & Aula \\
\hline \multirow[t]{8}{*}{ Controlabilidade } & $5,5 *(3,4)$ & Médio & $5,9 *(2,9)$ & $6,7^{*}(3,2)$ & $6,7 *(2,4)$ & $5,1 *(3,1)$ & $5,6 *(3,3)$ \\
\hline & & Alto & $4,6 *(3,2)$ & & & & \\
\hline & & Incontrolabilidade & Baixo & $9,0 \quad(1,8)$ & $6,9(2,2)$ & $6,2 \quad(2,9)$ & $8,0 \quad(2,2)$ \\
\hline & & & Médio & $7,1 \quad(2,1)$ & $7,1 \quad(2,1)$ & $5,2(3,1)$ & $7,3 \quad(2,3)$ \\
\hline & & & Alto & $5,9(3,2)$ & $6,4(2,4)$ & $5,1 \quad(3,1)$ & $4,3 \quad(2,1)$ \\
\hline & & Controlabilidade & Baixo & $8,6(2,2)$ & $7,6(2,4)$ & $6,8(2,6)$ & $7,7 \quad(2,7)$ \\
\hline & & & Médio & $6,0 \quad(2,7)$ & $5,5(2,9)$ & $3,8 \quad(3,2)$ & $4,7 \quad(2,9)$ \\
\hline & & & Alto & $3,5 \quad(3,2)$ & $6,6 \quad(2,3)$ & $3,8 \quad(3,0)$ & $2,1 \quad(3,1)$ \\
\hline
\end{tabular}

$* p \leq 0,001$

do grupo de custo baixo tenderam a informar maior intenção de ajuda $[F(2,528)=50,05 ; p \leq 0,001]$.

Quando considerada a interação entre as VIs, não se observou efeito interativo entre o tema e a atribuição $[F(3,527)$ $=1,75 ; \mathrm{ns}]$, o que indica que o conteúdo do tema não exerce um efeito relevante sobre a percepção de incontrolabilidade dos sujeitos, pois em todos os casos aqueles na condição de incontrolabilidade tenderam a ajudar mais. Em relação à interação entre o tema e o custo foi observada uma diferença significativa $[F(6,524)=38,07 ; p \leq 0,001]$, indicando que um custo mais baixo nem sempre esteve associado a maior intenção de ajuda. As variáveis independentes explicaram $28 \%$ da variabilidade da intenção de ajuda.

A mediação atribuição, emoções e ajuda foi testada por meio de análise de trajetórias, considerando os temas como variáveis moderadoras. A Figura 1 apresenta o modelo pictórico da análise de trajetórias. Portanto, esta análise foi feita para cada subamostra dos temas. $\mathrm{O}$ tema anotações (CFI = 0,93; NFI $=0,91)$ obteve os melhores indicadores de ajuste do modelo de mediação. Os temas fila $(\mathrm{CFI}=0,90 ; \mathrm{NFI}=0,87)$ e teatro $(\mathrm{CFI}=0,90 ; \mathrm{NFI}=0,86)$ obtiveram índices baixos de ajuste. $\mathrm{O}$ tema aula $(\mathrm{CFI}=0,68 ; \mathrm{NFI}=0,68)$ obteve índices ruins. Os coeficientes de regressão seguiram, conforme hipotetizado, uma relação negativa entre atribuição interna e emoções simpáticas e uma relação positiva entre esse último e a intenção de ajuda. Como pode ser observado na Tabela 2 , o único coeficiente que não foi significativo refere-se à relação entre atribuição interna e emoções simpáticas no tema teatro.

\section{Discussão}

O efeito de aspectos cognitivos na produção de estados 


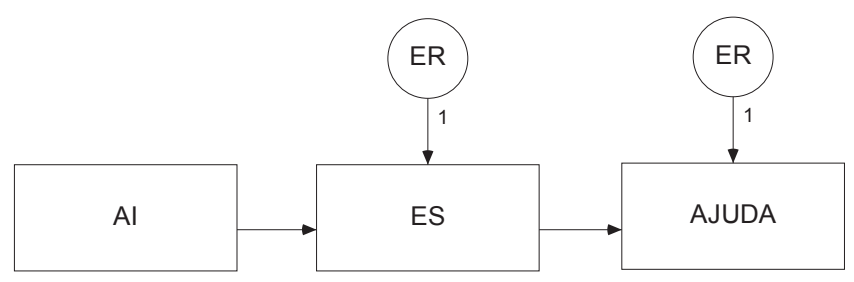

Figura 1. Modelo pictórico mediacional da relação atribuição-emoção-ajuda testado por meio de análise de trajetórias. AI - Atribuição Interna; ES - Emoções Simpáticas; ER Erro. O valor 1 refere-se a fixação dos parâmetros de regressão das variáveis observadas sobre seus respectivos erros.

Tabela 2

Coeficientes de determinação da análise de trajetórias em cada uma das sub-amostras dos temas dos cenários

\begin{tabular}{lcccc}
\hline Relações & Anotações & Teatro & Fila & Aula \\
\hline AI $\rightarrow$ ES & $-0,20(p=0,006)$ & $-0,11(p>0,05)$ & $-0,22(p=0,009)$ & $-0,27(p \leq 0,001)$ \\
ES $\rightarrow$ AJUDA & $0,46(p \leq 0,001)$ & $0,38(p \leq 0,001)$ & $0,29(p \leq 0,001)$ & $0,39(p \leq 0,001)$ \\
\hline
\end{tabular}

Legenda: AI - Atribuição Interna; ES - Emoções Simpáticas.

emocionais antecedentes do comportamento de ajuda continua sendo um fator relevante para ser investigado. A descrição de aspectos disposicionais e situacionais como antecedentes da avaliação que os indivíduos fazem dos que solicitam ajuda deve ser melhor explorada, para aumentar nossa compreensão da relação dos mesmos com emoções antecedentes do comportamento pró-social. Este trabalho apresentou evidências destas relações, corroborando o modelo teórico e ampliando o conjunto de evidências que o sustenta, pois foram produzidas evidências empíricas com o uso de novos cenários de simulação experimental.

De forma geral o modelo de Weiner (1980) foi corroborado, pois o efeito hipotetizado foi observado quando considerado como moderador o tema do cenário experimental. De forma geral as relações preditivas do modelo mediacional seguiram o esperado, pois quanto menor a atribuição de culpabilização maior a produção de emoções simpáticas em relação ao protagonista do cenário e maior a intenção de ajudar. Isto também foi corroborado pela ausência de significância na interação entre as VIs tema do cenário e atribuição na ANOVA.

$\mathrm{Na}$ análise de trajetórias apenas o tema do teatro não obteve uma relação significativa entre atribuição e emoções, mas a direção da relação ocorreu conforme o esperado. Considerando o menor número de participantes neste tema e um coeficiente de determinação de menor magnitude nesta relação o efeito foi apenas marginalmente significativo. Estes resultados corroboram o modelo de Weiner, como vários estudos já o fizeram (Betancourt, 1990; Meyer \& Mulherin, 1980; Reisenzein, 1986), mesmo com a inserção da variação da categorização social e da norma da situação emulada no cenário.

Um aspecto merece ser ressalvado no teste do modelo. $\mathrm{O}$ tema aula obteve um índice de ajuste consideravelmente ruim, bem abaixo dos demais temas. Isto demonstra que os dados não alcançaram os pressupostos de análise para a estimação por meio do método utilizado. Isto associado a um pequeno tamanho amostral resultou em um modelo com grande quantidade de resíduos, o que deve levar o leitor a avaliar com cuidado o teste do modelo mediacional para este tema especificamente.

A diferença em relação ao conteúdo dos temas foi relevante no concernente ao nível de custo pessoal. Como pode ser observado no resultado da ANOVA e nas médias para cada grupo experimental (Tabela 1), o custo foi avaliado como algo diferente em cada cenário. Por exemplo, no tema fila os participantes não diferenciaram o nível de custo pessoal nos níveis médio e alto, o que pode indicar que ter um compromisso ou parar em local proibido possui o mesmo nível de custo pessoal. Já no tema teatro o custo médio (não ter dinheiro para jantar após a peça) parece ter sido interpretado como algo pior do que não ter dinheiro para o resto da semana, pois o efeito previsto foi o inverso do esperado. Este tipo de ocorrência indica que a interpretação dos participantes para o custo variou conforme a situação emulada pelo cenário. Este efeito precisa ser mais bem investigado em outros estudos que avaliem o efeito do custo pessoal sobre o comportamento de ajuda, pois a dependência da interpretação do que seja custo pessoal deve ter uma relação estreita com o público da pesquisa e com a configuração da situação em que a ajuda é solicitada.

Aparentemente o efeito de favoritismo endogrupo não foi corroborado pelos dados empíricos deste estudo. Isto porque os temas que descreviam indivíduos próximos não tiveram uma tendência maior de intenção de ajuda. A literatura sobre processos grupais e comportamento pró-social tem apresentado evidências de que, em situações diversificadas, a categorização de exogrupo pode aumentar a chance de ajuda, o que contraria uma visão que inicialmente foi colocada sobre este fator, quando os processos grupais começaram a ser aplicados ao estudo do comportamento pró-social (Stürmer \& Snyder, 2010a). Vários fatores podem estar associados a esta ausência de favoritismo endogrupo no presente estudo. Sem dúvida um deles diz respeito a uma limitação metodológica do próprio estudo, pois não foi prevista uma estratégia para se avaliar o nível de identificação grupal que os sujeitos produziam em relação ao protagonista do 
cenário. De qualquer forma, é interessante seguir no processo de investigação da interação entre o processo de categorização social e a atribuição como um antecedente de emoções relativas ao comportamento pró-social. Isto porque estudos recentes apontam que a frequência de comportamento pró-social não é afetada pelo processo de categorização social, mas há fortes indícios de que o tipo de estado emocional despertado é distinto, pois os membros do endogrupo apresentam mais sentimentos de compaixão do que membros do exogrupo (Stürmer \& Snyder, 2010b). Esta é uma linha de investigação promissora para o estabelecimento de uma ligação entre dois diferentes níveis de análise, o que permitirá uma compreensão mais robusta dos elementos sociais que eliciam o comportamento pró-social.

Em relação à variação da emulação de normas sociais a partir dos temas dos cenários, o que se observa é que há um efeito desta variável sobre a intenção de ajuda, pois nos cenários que apresentam elementos mais salientes de reciprocidade (por exemplo, anotações e teatro) houve uma tendência maior de ajuda. Novamente este resultado deve ser interpretado com cuidado, pois o delineamento do estudo não previu uma forma de avaliar se os indivíduos assim perceberam as normas subjacentes aos cenários. De qualquer forma, estudos futuros devem considerar este aspecto como uma variável independente relevante para a produção de motivações pró-sociais.

De forma geral este estudo corrobora o modelo teórico de que a avaliação cognitiva que os indivíduos fazem sobre o comportamento exerce um efeito significativo para a produção de estados emocionais que antecedem o comportamento de ajuda, como previsto por Weiner $(1980,1995)$. Por outro lado o estudo também evidencia que há diferenças na interpretação que os indivíduos fazem dos cenários experimentais apresentados, pois a intenção de ajuda modifica-se significativamente a depender do cenário. Isto indica que um grande conjunto de fatores, já descritos na literatura de psicologia social, exerce um papel relevante na determinação do comportamento pró-social. Por este motivo é necessário avançar na compreensão do efeito que o processo de percepção social tem sobre a produção de estados emocionais antecedentes do comportamento pró-social.

A literatura em percepção social tem produzido novos modelos para se compreender a relação do processo atribucional com o comportamento social (Hilton, 2007). Malle (2004) apresenta um modelo importante, com base em pressupostos e formulações teóricas da teoria da mente (Sperb \& Maluf, 2008; Tomasello, Carpenter, Call, Behne, \& Moll, 2005). Em seu modelo Malle argumenta que modelos teóricos sobre atribuição, como é o caso do modelo de Weiner, tendem a tratar da mesma forma quatro aspectos da explicação do comportamento que são de natureza distinta. Malle postula que o comportamento intencional se diferencia significativamente, em termos de processos de percepção social, do comportamento não intencional. Os elementos estruturantes de seu modelo (por exemplo, causas, razões, histórias causais e fatores desencadeadores) se combinam para a construção de 'teorias populares' de explicação causal do comportamento.

A aproximação dos últimos desenvolvimentos teóricos das teorias atribucionais ao campo de investigação do comportamento pró-social é uma forma para se aprimorar a compreensão do efeito que os aspectos cognitivos possuem sobre a produção de estados emocionais pró-sociais. O problema da dicotomia presente na maioria dos modelos e da pesquisa em atribuição causal, na qual o comportamento é explicado ora por fatores disposicionais, ora por fatores situacionais, deixa de fora aspectos importantes de um processo que, como descrito por Malle (2004), carrega outras nuances. A literatura de psicologia social do comportamento pró-social ganharia bastante no desenvolvimento de um modelo teórico que contemplasse o conjunto de amplo espectro de combinações de fatores utilizado pelos indivíduos para explicar o comportamento de outros, o que nos permitiria desenvolver um conhecimento aprimorado sobre o efeito disto nos afetos antecedentes do comportamento pró-social.

\section{Agradecimentos}

O autor agradece as sugestões feitas pelo colega Fabio Iglesias e por dois revisores anônimos em versões prévias deste artigo. As sugestões resultaram na melhoria significativa do produto final. Este trabalho recebeu apoio no CNPq por meio de auxílio financeiro individual a pesquisador (Processo 400693/2008-9).

\section{Referências}

Aarts, H., Dijkesterhuis, A., \& Custers, R. (2003). Automatic normative behavior in environments: The moderating role of conformity in activating situational norms. Social Cognition, 21(6), 447-464.

Batson, C. D. (1998). Altruism and prosocial behavior. In D. T. Gilbert, S. T. Fiske, \& G. Lindzey (Orgs.), The handbook of social psychology (Vol. 2, pp. 282-316). New York: McGraw-Hill.

Batson, C. D. (2010). Empathy-Induced altruistic motivation. In M. Mikulincer \& W. R. Shadish (Orgs.), Prosocial motives, emotions, and behavior: the better angels of our nature (pp. 15-34). Washington: American Psychological Association.

Batson, C. D., van Lange, P. A. M., Ahmad, N., \& Lishner, D. L. (2003). Altruism and helping behavior. In M. Hogg \& J. Cooper (Orgs.), The SAGE handbook of social psychology (Vol. 1, pp. 279-295). London: SAGE.

Betancourt, H. (1990). An attribution-empathy model of helping behavior: behaviorial intentions and judgments of help giving. Personality and Social Psychology Bulletin, 16(3), 573-591.

Cialdini, R. B., Reno, R. R., \& Kallgren, C. A. (1990). A focus theory of normative conduct: recycling the concept of norms to reduce littering in public places. Journal of Personality and Social Psychology, 58(6), 1015-1026.

de Waal, F. B. M. (2008). Putting the altruism back intro altruism: The evolution of empathy. Annual Review of Psychology, 59, 279-300.

Dela-Coleta, J. A., \& Dela-Coleta, M. (2006). Atribuição de causalidade: teoria, pesquisa e aplicações. Taubaté: Cabral Editora e Livraria Universitária.

Dovidio, J. F., \& Penner, L. A. (2001). Helping and altruism. In G. J. O. Fletcher \& M. S. Clark (Orgs.), Blakwell handbook of social psychology: interpersonal processes (pp. 162-195). Oxford: Blackwell.

Dovidio, J. F., Piliavin, J. A., Schroeder, D. A., \& Penner, L. A. (2006). The social psychology of prosocial behavior. New York: Lawrence Earlbaum.

Eisenberg, N. (2010). Empathy-related responding: Links with self-regulation, moral judgements, and moral behavior. In M. Mikulincer \& P. R. Shaver (Orgs.), Prosocial motives, emotions, and behavior: the better angels of our nature (pp. 129-148). Washington: American Psychological Association.

Falcone, E. M. O., Ferreira, M. C., Luz, R. C. M., Fernandes, C. S., Faria, C. A., D'Augustin, J. F., ... Pinho, V. D. (2008). Inventário de Empatia (I.E.): Desenvolvimento e validação de uma medida brasileira. Avaliação 
Psicológica, 7(3), 321-334

Hair, J. F., Anderson, R. E., Tatham, R. L., \& Black, W. C. (2005). Análise Multivariada de Dados ( $5^{\mathrm{a}}$ ed.). Porto Alegre: Bookman.

Hein, G., \& Singer, T. (2010). Neuroscience meets social psychology: An integrative approach to human empathy and prosocial behavior. In M. Mikulincer \& P. R. Shaver(Orgs.), Prosocial motives, emotions and behavior: the better angels of our nature (pp. 109-125). Washington: American Psychological Association.

Hilton, D. (2007). Causal explanations: From social perception to knowledgebased causal attribution. In A. W. Kruglanski \& E. T. Higgins (Orgs.), Social psychology: handbook of basic principles (pp. 232-253). New York: The Guilford Press.

Koller, S. H., Camino, C., \& Ribeiro, J. (2001). Adaptação e validação interna de duas escalas de empatia para uso no Brasil. Estudos de Psicologia (Campinas), 18(3), 43-53.

Macrae, C. N., \& Johnston, L. (1998). Help, I need somebody: automatic action and inaction. Social Cognition, 16(4), 400-417.

Malle, B. F. (2004). How the mind explains behavior: folk explanations, meaning, and social interaction. Cambridge: Massachusetts Institute of Tecnology.

Meyer, J. P., \& Mulherin, A. (1980). From attribution to helping: an analysis of the mediating effects of affect and expectancy. Journal of Personality and Social Psychology, 39(2), 201-210.

Mikulincer, M., \& Shaver, P. R. (2010). Prosocial motives, emotions, and behavior: the better angels of our nature. Washington: American Psychological Association.

Moser, G., \& Corroyer, D. (2001). Politeness in the urban environment: is city life still synonymus with civility? Environment and Behavior, 33(5), 611-625.

Penner, L. A., Dovidio, J. F., Piliavin, J. A., \& Schroeder, D. A. (2005). Prosocial behavior: multilevel perspective. Annual Review of Psychology, 56(1), 365-392.

Pilati, R., Leão, M., Vieira, J. N., \& Fonseca, M. M. (2008). Efeitos da atribuição de causalidade e custo pessoal sobre a intenção de ajuda. Estudos de Psicologia, 13(3), 213-221. doi: 10.1590/S1413-294X2008000300004

Reisenzein, R. (1986). A structural equation analysis of Weiner's attribution-affect model of helping behavior. Journal of Personality and Social Psychology, 50(6), 1123-1133.

Rodrigues, A., \& Assmar, E. M. L. (2003). Influência social, atribuição de causalidade e julgamentos de responsabilidade e justiça. Psicologia: Reflexão e Crítica, 16(1), 191-201.

Sampaio, L. R., Camino, C. P. S., \& Roazzi, A. (2009). Revisão de aspectos conceituais, teóricos e metodológicos da empatia. Psicologia Ciência e Profissão, 29(2), 212-227.

Schmidt, G., \& Weiner, B. (1988). An attribution-afect-action theory of behavior: replications of judgments in help giving. Personality and Social Psychology Bulletin, 14(3), 610-621.

Shadish, W. R., Cook, T. D., \& Campbell, D. T. (2002). Experimental and quasiexperimental designs for generalized causal inference (Vol. 1). New York: Houghton Mifflin.

Sperb, T. M., \& Maluf, M. R. (2008). Desenvolvimento sociocognitivo: estudos brasileiros sobre teoria da mente. São Paulo: Vetor.

Stürmer, S., Snyder, M., \& Omoto, A. M. (2005). Prosocial emotions and helping: the moderating role of group membership. Journal of Personality and Social Psychology, 88(3), 532-546.

Stürmer, S., Snyder, M., Krop, A., \& Siem, B. (2006). Empathy-motivated helping: the moderating role of group membership. Personality and Social Psychology Bulletin, 32(7), 943-956.

Stürmer, S., \& Snyder, M. (2010a). The psychological study of group processes and intergroup relations in prosocial behavior: past, Present, and Future. In S. Stürmer \& M. Snyder (Orgs.), The psychology of prosocial behavior (pp. 3-10). Oxford: Wiley-Blackwell.

Stürmer, S., \& Snyder, M. (2010b). Helping 'us'versus 'them': Towards a grouplevel theory of helping and altruism within and across group boundaries. In S. Stürmer \& M. Snyder (Orgs.), The psychology of prosocial behavior (pp. 13-32). Oxford: Wiley-Blackwell.

Tabachnick, B., \& Fidell, L. S. (2001). Using multivariate statistics. San Francisco: Allyn \& Bacon.

Tomasello, M., Carpenter, M., Call, J., Behne, T., \& Moll, H. (2005). Understanding and sharing intentions: the origins of cultural cognition. Behaviorial and Brain Sciences, 28, 675-735.

Weiner, B. (1980). A cognitive (attribution) - Emotion - Action model of motivated behavior: an analysis of judgments of help-giving. Journal of Personality and Social Psychology, 39(2), 186-200.

Weiner, B. (1995). Judgments of responsability: a foundation for a theory of social conduct. New York: Guilford.

Ronaldo Pilati, Doutor em Psicologia pela Universidade de Brasília, é professor Adjunto na mesma universidade. Endereço para correspondência: Departamento de Psicologia Social e do Trabalho, Sala AT013, ICC/Sul, Campus Universitário Darcy Ribeiro, Asa Norte, Brasília/DF. CEP: 70.910-900. Fone/Fax: (61)3107-6828. E-mail: rpilati@gmail.com 